\title{
Topologically-mediated energy release by relativistic antiferromagnetic solitons
}

\author{
R. M. Otxoa,,${ }^{1,2,}{ }^{*}$ R. Rama-Eiroa $\odot,{ }^{2,3}$ P. E. Roy, ${ }^{1}$ G. Tatara, ${ }^{4}$ O. Chubykalo-Fesenko, ${ }^{5}$ and U. Atxitia ${ }^{6}$ \\ ${ }^{1}$ Hitachi Cambridge Laboratory, J. J. Thomson Avenue, Cambridge CB3 OHE, United Kingdom \\ ${ }^{2}$ Donostia International Physics Center, 20018 San Sebastián, Spain \\ ${ }^{3}$ Polymers and Advanced Materials Department: Physics, Chemistry, and Technology, \\ University of the Basque Country, UPV/EHU, 20018 San Sebastian, Spain \\ ${ }^{4}$ RIKEN Center for Emergent Matter Science (CEMS) and RIKEN Cluster for Pioneering Research (CPR), \\ 2-1 Hirosawa, Wako, Saitama 351-0198, Japan \\ ${ }^{5}$ Instituto de Ciencia de Materiales de Madrid, CSIC, Cantoblanco, 28049 Madrid, Spain \\ ${ }^{6}$ Dahlem Center for Complex Quantum Systems and Fachbereich Physik, Freie Universitat Berlin, 14195 Berlin, Germany
}

(Received 1 July 2021; revised 23 September 2021; accepted 29 September 2021; published 25 October 2021)

\begin{abstract}
Magnetic solitons offer functionalities as information carriers in multiple spintronic and magnonic applications. However, their potential for nanoscale energy transport has not been revealed. Here we demonstrate that antiferromagnetic solitons, e.g., domain walls, can uptake, transport, and release energy. The key for this functionality resides in their relativistic kinematics; their self-energy increases with velocity due to Lorentz contraction of the soliton and their dynamics can be accelerated up to the effective speed of light of the magnetic medium. Furthermore, their classification in robust topological classes allows us to selectively release this energy back into the medium by colliding solitons with opposite topology. Our work uncovers important energy-related aspects of the physics of antiferromagnetic solitons and opens up the attractive possibility for spin-based nanoscale and ultrafast energy transport devices.
\end{abstract}

DOI: 10.1103/PhysRevResearch.3.043069

\section{INTRODUCTION}

Solutions for an efficient control of energy in nanoelectronics are based on identifying the prevailing carriers and transfer mechanisms of energy at relevant time and length scales. A plethora of concepts based on the electron properties have been proposed for efficient transport and release of energy at the nanoscale [1]. Spin-based devices offer additional functionalities for nanoelectronics [2]. Magnonic devices based on the collective spin wave properties are proposed as low dissipation components which can enable compact electronics in the future [3]. Particularly, they offer efficient information transport and are promising for nontraditional computation architectures. Localized spin structures have also been discussed broadly as information carriers [4,5] or more rarely as stationary energy storing devices [6-9], however the possibility of their use as energy carriers is barely known.

A well-determined free energy is stored in localized magnetic textures-noncollinear spin structures-such as domain walls (DWs), vortices, and skyrmions, whose stability is grounded to their nontrivial topology [10-13]. These magnetic solitons (MSs) already play a pivotal role for the

\footnotetext{
*ro274@cam.ac.uk
}

Published by the American Physical Society under the terms of the Creative Commons Attribution 4.0 International license. Further distribution of this work must maintain attribution to the author(s) and the published article's title, journal citation, and DOI. development of spin-based applications such as processing [4,5], sensing [14], storing information [15], as well as radio-frequency [16] and neuroinspired devices $[17,18]$. The simplest example of a MS is the DW, which separates magnetic domains magnetized in opposite directions [19]. The exchange energy stored in the DW can be transported by moving the DW with magnetic fields [20], spin currents [21,22], spin waves [23-25], or even thermal gradients [26,27]. The only way to change the energy of a DW is by controlling its width. As the angle between neighboring atomic spin increases (corresponding to a width reduction), the exchange energy arising from their interaction increases.

Thus, efficient and ultrafast means for loading, transporting, and extracting the free energy at magnetic textures are missing. Our proposal relies on the unique dynamical properties of DWs (MSs) in antiferromagnetic (AFM) materials [28-32]. Since they obey the relativistic kinematics, their width and energy strongly depend on the soliton velocity [Fig. 1(a)], which results in a significant increase of magnetic energy in the system. Differently to DWs in ferromagnets, which are prone to deformation at relatively low velocities [19,33-36], AFM DWs offer the possibility to transport their self-energy at speeds close to the effective speed of light of the medium $c$ [37] [Fig. 1(a)]. As they preserve their shape through time, these MSs enable long-range coherent energy transport $[38,39]$. Notably, since the relative orientation of atomic spins within the DWs can be classified into two distinct topological classes [Fig. 1(b)], here we demonstrate the possibility of topologically-mediated energy release by collision of two relativistic AFM DWs [Figs. 1(c) and 1(d)]. In AFMs, the 
(a)

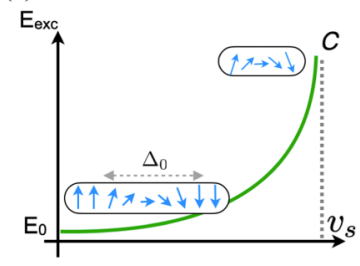

(b)

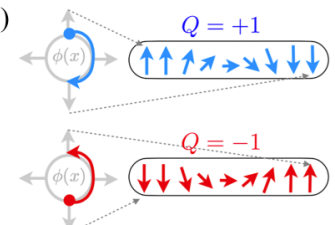

(c)

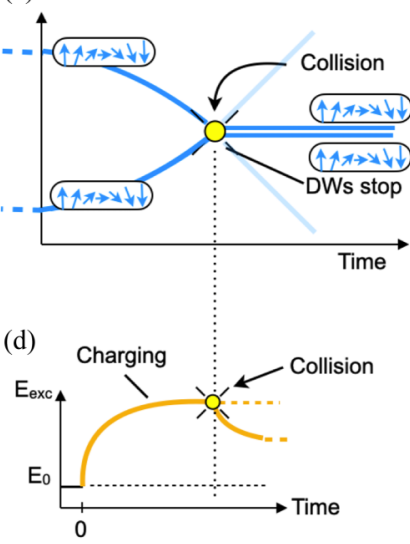

(e)

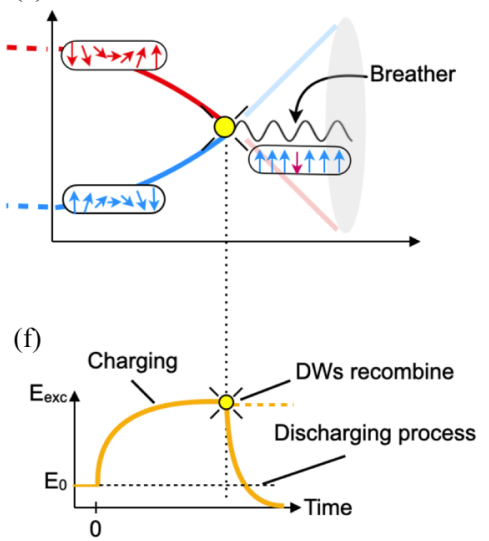

FIG. 1. (a) A magnetic domain wall (DW) is a region of width $\Delta_{0}$ at rest, which separates two magnetic domains. In AFMs DWs can be driven up to relativistic speeds $v_{s}$ resulting in the Lorentz contraction of the DW, $\Delta=\Delta_{0} \gamma_{\mathrm{L}}$, and the increase of the self-energy, $E\left(v_{s}\right)=E_{0} / \gamma_{\mathrm{L}}$, where $\gamma_{\mathrm{L}}=\sqrt{1-\left(v_{s} / c\right)^{2}}$. (b) DWs can hold two spin winding numbers $Q= \pm 1$, which are degenerated in energy. The winding number is defined by the integral over the $x$ axis of the topological density, $-\nabla_{x} \phi(x)$, where $\phi(x)$ is the magnetization azimuthal (in-plane) angle and $x$ is the DW propagation direction. For $Q=1$, at $x \rightarrow-\infty$, starting at $\phi=\pi / 2$, the path (blue arrow) to $\infty, \phi=-\pi / 2$ defines its topology. For $Q=-1, \phi(-\infty)=-\pi / 2$ and $\phi(\infty)=\pi / 2$. (c) Schematic representation of two DWs with the same winding number, $Q=+1$ and $Q=+1$, approaching each other in an accelerated motion until collision. After collision DWs stop. (d) During the motion of the DWs the self-energy increases due to Lorentz contraction caused by the increase of their speed. After collision, the self-energy stored at the DWs is partially discharged. (e) Schematic representation of two DWs with the opposite winding number, $Q=+1$ and $Q=-1$, approaching each other in an accelerated motion until collision. After collision DWs annihilate and a breather appears. (f) During the motion of the DWs the self-energy rises due to Lorentz contraction caused by the increase of their speed. After collision, the self-energy stored at the DWs is completely discharged.

role played by the photons in special relativity corresponds to the magnons, and $c$ corresponds to their maximum group velocity. A direct consequence of special relativity is that the MSs' width at rest, $\Delta_{0}$, contracts as its velocity $v_{\mathrm{s}} \rightarrow c$, described by Lorentz factor $\gamma_{\mathrm{L}} ; \Delta=\gamma_{\mathrm{L}} \Delta_{0}$, where $\gamma_{\mathrm{L}}\left(v_{\mathrm{S}}\right)=$ $\sqrt{1-\left(v_{\mathrm{s}} / c\right)^{2}}$. An immediate consequence is that MSs' energy can also be represented in a relativistic form given by

$$
E_{\mathrm{exc}}\left(v_{\mathrm{s}}\right)=\frac{E_{0}}{\sqrt{1-\left(v_{\mathrm{s}} / c\right)^{2}}},
$$

where $E_{0}$ corresponds to the MSs' energy at rest [Fig. 1(a)].

Thus, the relativistic nature of AFM solitons offers the possibility to "charge" them by just increasing their velocity. This energy uptake process is considerably fast since the AFM soliton reaches its maximum velocity energy in only a few picoseconds due to its small inertia [27] allowing for ultrafast optical excitation $[40,41]$. Theoretically at those speeds the DW can contract down to a few nanometers [42] [Fig. 1(a)]. Importantly, relativistic soliton physics is not only a theoretical construct, but indirect experimental verification has been recently achieved in ferrimagnetic insulators [43]. Although propagation at relativistic velocities of individual AFM DWs has been investigated theoretically, their interactions with each other and the role of topology remain unexplored.

A continuous deformation of the order parameter into a different topological class is by principle impossible in infinitely extended films [44] and difficult in confined geometries with sizes larger than the characteristic exchange correlation length. Regarding energy release, access to the energy transported by the MSs is only possible via topologically conserved processes, namely, for those where the so-called topological charge (winding number) $Q$ of the magnetic medium is conserved. For instance, for a one-dimensional (1D) DW, the topological charge is defined as the integral over the space of the winding number density, $w(x, t)=$ $-\nabla_{x} \phi(x, t)$. Here $\phi(x, t)$ is the in-plane angle of the spin at location $x$ at time $t$ of the spin configuration along the 1D line of the propagation direction along the $x$ axis [Fig. 1(b)]. The total winding number or topological charge is expressed as: $Q=\frac{1}{\pi} \int w(x, t) d x$. Therefore, a DW in a system can exist with only two distinct topological flavors, $Q= \pm 1$ [Fig. 1(b)]. It is important to note that the homogeneous state corresponds to $Q_{0}=0$ since $\phi(x, t)=$ constant. This means that one DW $(Q= \pm 1)$ would not decay into the ground energy state $\left(Q_{0}=\right.$ $0)$ even though it might correspond to a lower energy level (when thermal activation processes could be neglected). However, when two DWs are present in the system two scenarios are possible. When two DWs of the same topology $\left(Q_{1}=+1\right.$ and $Q_{2}=+1$ ) are driven to collide, they cannot collapse into an homogeneous state [Fig. 1(c)]. Transfer of their energy into the system is only partial, and most of it remains within the spin system [Fig. 1(d)]. However, when at least two DWs with opposite topological charge $\left(Q_{1}=+1\right.$ and $\left.Q_{2}=-1\right)$ are forced to collide [Fig. 1(e)], a recombination process is accessible both from energy and topological arguments as now $Q_{0}=Q_{1}+Q_{2}=0$. In such a case, the DWs recombine [Fig. 1(f)], and the energy at the DWs is completely accessible.

Since the stability of a MS and the output of their collisions are rooted in topological arguments, from now on we refer to a MS as a topological magnetic soliton (TMS). In this work we show that AFM solitons can be "loaded" by magnetic energy, transport, and release. We pay special attention to the energy release process, by investigating the outcome of the collision of two high-energy AFM DWs. Our results unambiguously 
demonstrate the role of topology and relativistic nature of AFM TMS in these processes.

\section{COLLISION OF TWO DOMAIN WALLS}

\section{Self-energy and interaction energy}

To describe 1D magnetic DWs, it is convenient to introduce the Walker-like symmetric rigid profile, given by

$$
\phi_{i}(x, t)=2 \arctan \exp \left[\frac{Q_{i}\left(x-X_{i}(t)\right)}{\Delta}\right],
$$

the expression of which is characterized by the DW topological charge $Q_{i}$, the central position of the soliton $X_{i}$, and its DW width $\Delta[20,39]$. We consider a system consisting of two DWs, denoted through $i=1,2$ indices, with DW topological charges and central positions $Q_{1,2}$ and $X_{1,2}$, respectively. In this case, the combined profile of the system is given by

$$
\begin{aligned}
\phi(x, t)= & \sum_{i} \phi_{i}(x, t)=2 \arctan \exp \left[\frac{Q_{1}\left(x-X_{1}(t)\right)}{\Delta}\right] \\
& +2 \arctan \exp \left[\frac{Q_{2}\left(x-X_{2}(t)\right)}{\Delta}\right] .
\end{aligned}
$$

The total exchange energy, $E_{\text {exc }}$, composed of both magnetic textures for the case of a 1D spin chain, can be expressed as

$$
E_{\mathrm{exc}}=a_{0}^{2} A \int_{-\infty}^{+\infty}\left(\sum_{i} \partial_{x} \phi_{i}(x, t)\right)^{2} d x,
$$

where $A$ represents the effective exchange stiffness of the system, $a_{0}$ is the in-plane lattice constant [32], and where $\partial_{x}$ expresses the spatial derivative along the $x$ th spatial direction of the profile of each DW, the latter being given by

$$
\partial_{x} \phi_{i}(x, t)=\frac{Q_{i}}{\Delta} \operatorname{sech}\left(\frac{x-X_{i}(t)}{\Delta}\right) .
$$

Taking into account Eq. (5), it is possible to explicitly write the terms inside the integral of Eq. (4) as follows:

$$
\begin{aligned}
& \left(\sum_{i} \partial_{x} \phi_{i}(x, t)\right)^{2} \\
& =\frac{1}{\Delta^{2}}\left[\operatorname{sech}^{2}\left(\frac{x-X_{1}(t)}{\Delta}\right)+\operatorname{sech}^{2}\left(\frac{x-X_{2}(t)}{\Delta}\right)\right. \\
& \left.\quad+2 Q_{1} Q_{2} \operatorname{sech}\left(\frac{x-X_{1}(t)}{\Delta}\right) \operatorname{sech}\left(\frac{x-X_{2}(t)}{\Delta}\right)\right] .
\end{aligned}
$$

Substituting in Eq. (4), it is possible to identify different exchange-based contributions to the system. Each DW selfenergy $E_{\mathrm{exc}}^{i}$ is therefore given by

$$
E_{\mathrm{exc}}^{i}=\frac{a_{0}^{2} A}{\Delta^{2}} \int_{-\infty}^{+\infty} \operatorname{sech}^{2}\left(\frac{x-X_{i}(t)}{\Delta}\right) d x .
$$

An additional energy contribution comes from the exchange interaction energy between both DWs, $E_{\text {exc }}^{1,2}$, which is expressed as

$$
E_{\mathrm{exc}}^{1,2}=\frac{2 Q_{1} Q_{2} a_{0}^{2} A}{\Delta^{2}} \int_{-\infty}^{+\infty} \operatorname{sech}\left(\frac{x-X_{1}(t)}{\Delta}\right)
$$

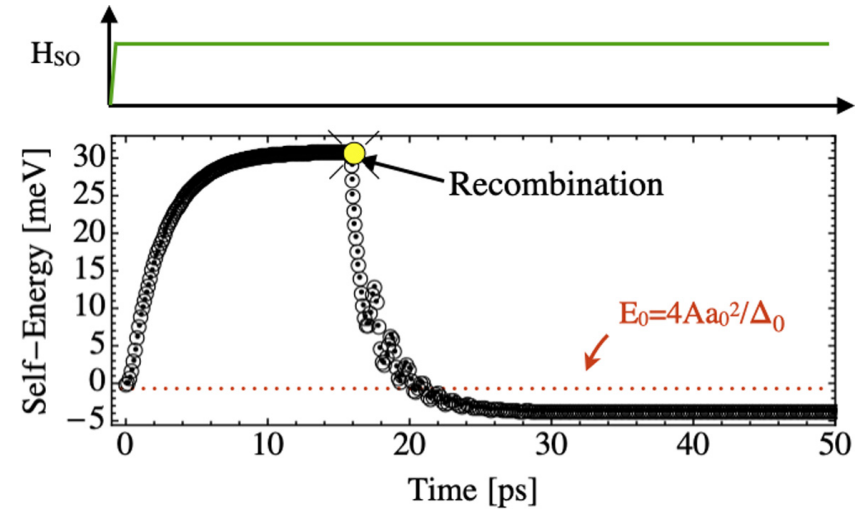

FIG. 2. Time dependence of the self-energy of the two antiferromagnetic domain walls (DWs) when subject to a spin-orbit field $H_{\mathrm{SO}}$, which ramps up from $H_{\mathrm{SO}}=0$ to $H_{\mathrm{SO}}=60 \mathrm{mT}$ in $10 \mathrm{fs}$, in the case of two solitons with opposite topological charges, which entails their annihilation when they are conducted towards each other, which implies the liberation of the self-energy of both textures. The red dashed line represents the sum of the static self-energy of the two DWs, $E_{0}=2 E_{\text {exc }}^{i}\left(H_{\text {SO }}=0\right)=4 a_{0}^{2} A / \Delta_{0}$, where $A$ represents the effective exchange stiffness of the medium, $a_{0}$ is the in-plane lattice constant, and $\Delta_{0}$ expresses the DW width at rest, according to Eq. (9), which is taken as a zero reference for the time evolution of the exchange energy of the system.

$$
\times \operatorname{sech}\left(\frac{x-X_{2}(t)}{\Delta}\right) d x,
$$

which allows for writing the total energy $E_{\text {exc }}$ as the sum of the following independent exchange contributions: $E_{\text {exc }}=$ $E_{\mathrm{exc}}^{1}+E_{\mathrm{exc}}^{2}+E_{\mathrm{exc}}^{1,2}$. Analyzing the individual contributions to the exchange energy by the magnetic textures, expressed in Eq. (7), it is possible to obtain that

$$
E_{\mathrm{exc}}^{i}=\frac{2 a_{0}^{2} A}{\Delta},
$$

so if the self-energy of both textures is taken into account, we can see that, regardless of their topological charge $Q_{i}$, both contributions will be equal, giving rise to $E_{\mathrm{exc}}^{1}+E_{\mathrm{exc}}^{2}=$ $2 E_{\text {exc }}^{i}=4 a_{0}^{2} A / \Delta$, since the involved hyperbolic function is an even function. The time evolution of the self-energy $E_{\text {exc }}^{i}$ as a function of the relativistic contraction when subject to a spinorbit (SO) field $H_{\text {SO }}$ is shown in Fig. 2. Therefore, a DW has a self-energy that is defined by intrinsic material dependent magnetic parameters, such as exchange and anisotropy energy terms. Therefore, no clear mechanism can be envisioned to selectively modify on demand the energy stored in DWs without changing their intrinsic magnetic parameters.

\section{RESULTS AND DISCUSSION}

\section{A. Simulations of the collision of two domain walls}

We quantify and determine the energy flow dynamics associated to the DW motion and the collision of two high energy DWs in the AFM metal $\mathrm{Mn}_{2} \mathrm{Au}$. This material presents a high Néel temperature of circa $1500 \mathrm{~K}$ [45] and an efficient electric control of the DW motion $[32,42,46]$. Upon passing an electrical current along the basal planes, the so-called inverse spin galvanic effect [47] produces a staggered local spin 
TABLE I. Literature values for material parameters relevant for modeling the spin dynamics $[45,50] . k_{\mathrm{B}}$ is Boltzmann's constant.

\begin{tabular}{lcccccc}
\hline \hline $\begin{array}{l}J_{i 1} k_{\mathrm{B}}^{-1} \\
(\mathrm{~K})\end{array}$ & $\begin{array}{c}J_{i 2} k_{\mathrm{B}}^{-1} \\
(\mathrm{~K})\end{array}$ & $\begin{array}{c}J_{i 3} k_{\mathrm{B}}^{-1} \\
(\mathrm{~K})\end{array}$ & $\begin{array}{c}K_{2 \perp} \\
(\mathrm{J})\end{array}$ & $\begin{array}{c}K_{2 \|} \\
(\mathrm{J})\end{array}$ & $\begin{array}{c}K_{4 \perp} \\
(\mathrm{J})\end{array}$ & $\begin{array}{c}K_{4 \|} \\
(\mathrm{J})\end{array}$ \\
\hline-396 & -532 & 115 & $-1.303 \times 10^{-22}$ & $7 K_{4 \|}$ & $2 K_{4 \|}$ & $1.855 \times 10^{-25}$ \\
\hline \hline
\end{tabular}

accumulation with opposite polarities in each sublattice which creates a local staggered spin-orbit (SO) field perpendicular to the current direction. The dynamics of a DW induced by SO torques can be described by the Landau-Lifshitz-Gilbert (LLG) equation for the atomistic spin dynamics.

We perform atomistic spin dynamics simulations for the full $\mathrm{Mn}_{2} \mathrm{Au}$ crystal structure. A unit cell is replicated along the $x$ direction 6000 times representing circa $2 \mu \mathrm{m}$ of physical spin space. The system has periodic boundary conditions imposed along the $y$ direction while open boundaries are considered along the $x$ and $z$ directions. The configuration energy is constituted by three exchange interactions (two antiferromagnetic and one ferromagnetic), magnetocrystalline energy contributions, and the SO field (Supplemental Material [48]). The total energy $E$ is:

$$
\begin{aligned}
E= & -2 \sum_{\langle i<j\rangle} J_{i j} \mathbf{S}_{i} \cdot \mathbf{S}_{j}-K_{2 \perp} \sum_{i}\left(\mathbf{S}_{i} \cdot \hat{\mathbf{z}}\right)^{2}-K_{2 \|} \sum_{i}\left(\mathbf{S}_{i} \cdot \hat{\mathbf{y}}\right)^{2} \\
& -\frac{K_{4 \perp}}{2} \sum_{i}\left(\mathbf{S}_{i} \cdot \hat{\mathbf{z}}\right)^{4}-\frac{K_{4 \|}}{2} \sum_{i}\left[\left(\mathbf{S}_{i} \cdot \hat{\mathbf{u}}_{1}\right)^{4}+\left(\mathbf{S}_{i} \cdot \hat{\mathbf{u}}_{2}\right)^{4}\right] \\
& -\mu_{0} \mu_{\mathrm{s}} \sum_{i} \mathbf{S}_{i} \cdot \mathbf{H}_{i}^{\text {so }} .
\end{aligned}
$$

The first term on the right-hand side is the exchange energy where $J_{i j}$ is the exchange coefficient along the considered bonds $[42,49]$. The second and third terms are the uniaxial hard and easy anisotropies of strengths $K_{2 \perp}$ and $K_{2 \|}$, respectively, while the fourth and fifth terms collectively describe tetragonal anisotropy. For the in-plane part of the tetragonal anisotropy, $\mathbf{u}_{1}=[110]$ and $\mathbf{u}_{2}=[1 \overline{1} 0]$. Finally, $\mu_{0}$ and $\mu_{\mathrm{s}}$ are the magnetic permeability in vacuum and the magnetic moment, respectively, and $\mathbf{H}_{i}^{\text {so }}$ is the SO field applied at each atomic site. We have used $\mu_{\mathrm{s}}=4 \mu_{B}$ [45], with $\mu_{B}$ being the Bohr magneton. The time evolution of a unit vector spin at site $i, \mathbf{S}_{i}$, is simulated by solving the Landau-Lifshitz-Gilbert equation:

$$
\frac{d \mathbf{S}_{i}}{d t}=-\gamma \mathbf{S}_{i} \times \mathbf{H}_{i}^{\mathrm{eff}}-\gamma \alpha_{\mathrm{G}} \mathbf{S}_{i} \times\left(\mathbf{S}_{i} \times \mathbf{H}_{i}^{\mathrm{eff}}\right),
$$

where $\gamma$ is the gyromagnetic ratio of a free electron $(2.21 \times$ $\left.10^{5} \mathrm{~m} / \mathrm{As}\right), \alpha_{\mathrm{G}}$ is the Gilbert damping set here to 0.001 , and $\mathbf{H}_{i}^{\text {eff }}$ is the effective field evaluated at each spin site in time using Eq. (10) as $\mathbf{H}_{i}^{\text {eff }}=\frac{-1}{\mu_{0} \mu_{\mathrm{s}}} \frac{\delta E}{\delta \mathbf{S}_{i}}$. The system of equations, Eq. (11), is solved by a fifth order Runge-Kutta method. Material constants used are summarized in Table I.

To follow the motion of a DW in a stripe with a long dimension parallel to the $x$ axis, it is sufficient to monitor the $m_{x}$ projection of the magnetization along the track. The initial condition in our computational model corresponds to two DWs separated by approximately $1.5 \mu \mathrm{m}$. The SO field generated by the laterally injected electrical current acts onto
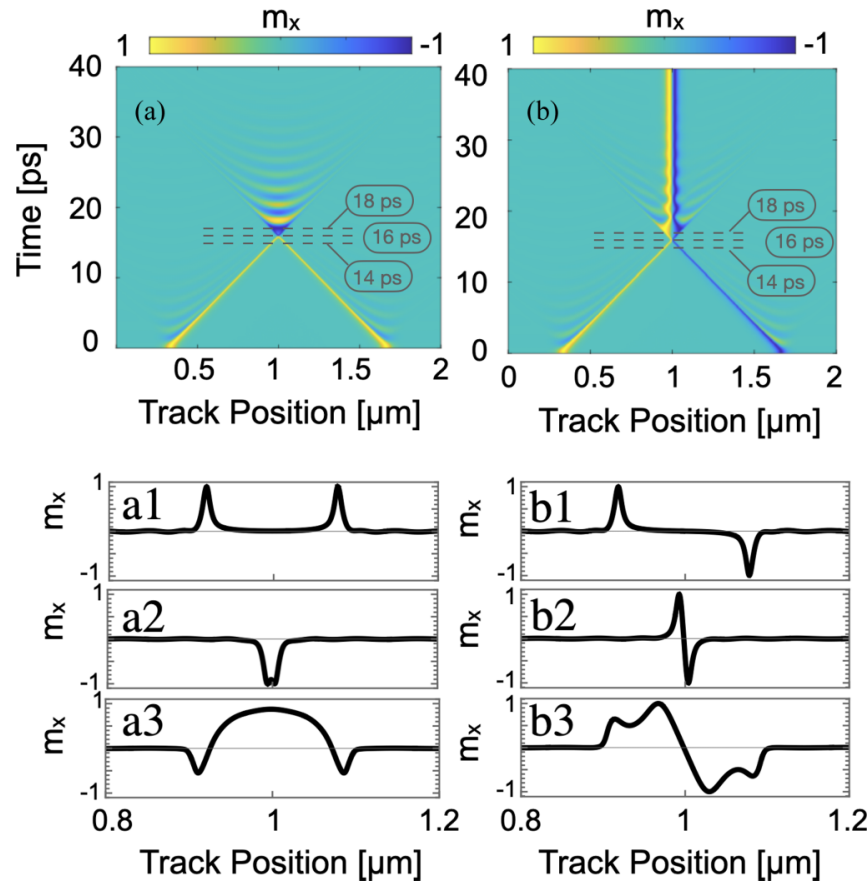

FIG. 3. Model simulations of the collision of two domain walls (DWs) in $\mathrm{Mn}_{2} \mathrm{Au}$. (a),(b) Spatiotemporal diagram of the dynamics of the process showing the DWs collision and the corresponding output. (a) Two DWs with opposite topological charges result after the collision in a dispersing breather. (b) Two DWs with the same topological charges after the collision stop and increase their size. A finite $x$ component of the magnetization, $m_{x}$, represents the position and extension of the DWs. Subplots (a1),(b1), (a2),(b2), and (a3),(b3) are cuts of the color map in (a) and (b) for three characteristic times $t=14,16$, and $18 \mathrm{ps}$, respectively.

the DWs through the Zeeman energy. In order to reduce the Zeeman energy, the magnetic domain between the two DWs shrinks, moving both DWs towards each other. The DWs reach their final velocities of around $42.4 \mathrm{~km} / \mathrm{s}$ for a $S O$ field of $60 \mathrm{mT}$ with a ramping time of $10 \mathrm{fs}$ in only a couple of picoseconds. This speed constitutes $\sim 98 \%$ of the maximum magnon velocity $c$ for $\mathrm{Mn}_{2} \mathrm{Au}$. For the range of $\mathrm{SO}$ fields investigated, the DW width reduces from $20 \mathrm{~nm}$ to $4 \mathrm{~nm}$ due to the Lorentz contraction. This leads to an increase of a $500 \%$ of the DWs energy which can be transported [Figs. 2 and 1(a)]. Notably, DWs are very stable with no visible spin-wave generation at these high velocities. Circa 15 ps later both DWs reach each other [Figs. 3(a) and 3(b)] and collide. Two scenarios arise depending upon the topological charge carried by them and topology conservation laws.

Specifically, when $Q_{1}=-Q_{2}=1$, topological charge conservation rules allow the DWs to recombine leading to a homogeneous state $Q_{0}=0$ [Fig. 3(a)]. At an instant prior to the collision ( $t=14 \mathrm{ps}$ ), both DWs are well defined [Fig. 3(a1)], close to the moment at which they annihilate each other ( $t=16 \mathrm{ps})$ the two DW profiles merge [Fig. 3(a2)], and some time after their disappearance as individual entities ( $t=18 \mathrm{ps}$ ), a bounded dispersing stationary breather mode is observed [Fig. 3(a3)].

Interestingly, a breather mode [39] is created out of the collision between the two solitons, which is localized in the space 
time by a cone defined by the trajectory of each DW before the collision [Fig. 1(e)]. No spin perturbations can exist outside this cone. We observe that the past magnon cone behaves like the future magnon cone in reverse, however, differently to relativity, spin perturbations lie behind the DW motion, namely outside the cone [51]. Importantly, the breather mode attenuation time is given by the exchange relaxation time scale: $2 \alpha_{\mathrm{G}} \gamma J_{\mathrm{AFM}} / \mu_{\mathrm{s}} \approx 3-4$ ps. In layered $\mathrm{AFM}$, such as $\mathrm{Mn}_{2} \mathrm{Au}, J_{\mathrm{AFM}}$ represents the effective exchange interaction between layers. This sets an ultrafast discharge timescale. For more details of the dynamics see also Supplemental Material [48].

When $Q_{1}=Q_{2}=1$, topological charge conservation rules do not allow for DWs to recombine leading to the homogeneous state $\left(Q_{0}=0\right)$. Similar to the previous scenario, at an instant prior to the collision ( $t=14 \mathrm{ps})$, both DWs are well defined [Fig. 3(b1)]. After the collision, the DWs stop their rectilinear motion (conservation of momentum) $(t=16 \mathrm{ps})$ [Fig. 3(b2)] hence the Lorentz factor $\gamma_{\mathrm{L}} \approx 1$, and as a consequence the DWs, widen almost instantaneously but start to oscillate around their final position emitting spin waves $(t=18$ ps) [Fig. 3(b3)]. The final state, i.e., DW widths and separation, however, depends on energetic considerations.

\section{B. Stationary state of the two domain walls $\left(Q_{1}=Q_{2}\right)$ after collision}

One can calculate the energy of the couple of DWs by solving the integral given by Eq. (8), which represents the DW interaction, and is more complicated to solve than the other two terms corresponding to the self-energy. To solve it, we use the following trigonometric relationship

$$
\cosh \left(\xi_{1}\right) \cosh \left(\xi_{2}\right)=a+a \cosh \left(2 \xi_{1}\right)+b \sinh \left(2 \xi_{1}\right) \text {, }
$$

where $\xi_{i}=\left(x-X_{i}(t)\right) / \Delta$, and

$$
\begin{aligned}
& a=\frac{1}{2} \cosh \left(\frac{X_{1}(t)-X_{2}(t)}{\Delta}\right), \\
& b=\frac{1}{2} \sinh \left(\frac{X_{1}(t)-X_{2}(t)}{\Delta}\right) .
\end{aligned}
$$

The integral in Eq. (8) reduces to

$$
\begin{aligned}
E_{\mathrm{exc}}^{1,2} & =\frac{2 Q_{1} Q_{2} a_{0}^{2} A}{\Delta^{2}} \int_{-\infty}^{+\infty} \frac{d x}{a+a \cosh \left(2 \xi_{1}\right)+b \sinh \left(2 \xi_{1}\right)} \\
& =\frac{2 Q_{1} Q_{2} a_{0}^{2} A}{\Delta^{2}} I,
\end{aligned}
$$

where the integral $I$ can be rewritten as the following sum of terms

$$
\begin{aligned}
I= & \frac{\Delta}{2} \int_{0}^{+\infty}\left[\frac{d \xi}{a+a \cosh (\xi)+b \sinh (\xi)}\right. \\
& \left.+\frac{d \xi}{a+a \cosh (\xi)-b \sinh (\xi)}\right] \\
= & \frac{\Delta}{2}[I(a, a, b)+I(a, a,-b)] .
\end{aligned}
$$

This integral has a tabulated solution [52], given by

$$
I(a, a, \pm b)= \pm \frac{1}{b} \ln \left(\frac{a \pm b}{a}\right) .
$$

(a)

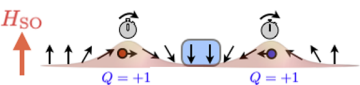

(b)

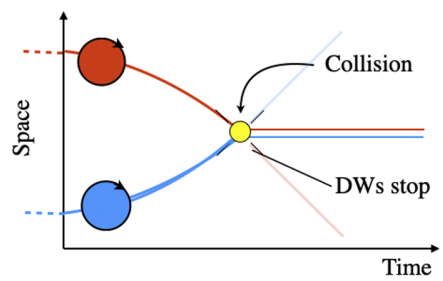

(c)

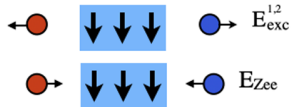

(d)

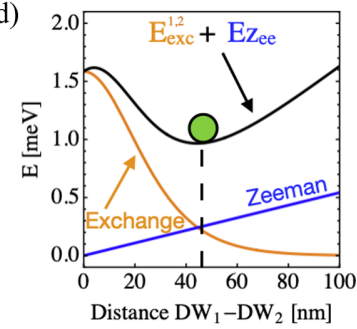

(e)

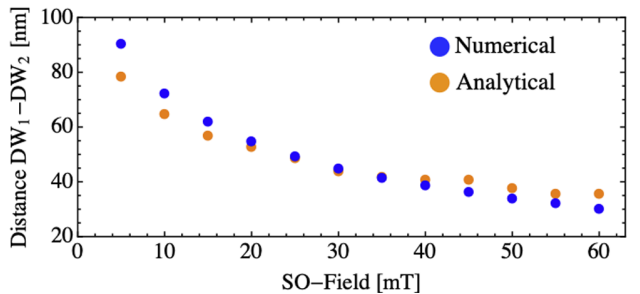

FIG. 4. (a) Sketch of two domain walls (DWs) with the same chirality $Q=+1$ under the action of an SO field $H_{\mathrm{SO}}$. They move driven by SO field antiparallel to the central magnetic domain (blue box). (b) DWs move until collision. After the collision, both DWs remain at an equilibrium distance from each other. (c) Schematic illustration of the role played by the exchange $E_{\mathrm{exc}}^{1,2}$ and Zeeman $E_{\mathrm{Zee}}$ energies. While the exchange energy tries to separate $\mathrm{DW}_{1}$ and $\mathrm{DW}_{2}$, the Zeeman energy forces them to stay as close as possible. (d) Zeeman (blue line) and exchange (orange line) energies as a function of the relative distance between $\mathrm{DW}_{1}$ and $\mathrm{DW}_{2}$ for an applied SO field of $20 \mathrm{mT}$. (e) Comparison between analytical Eq. (21) and numerically extracted stable distances between $\mathrm{DW}_{1}$ and $\mathrm{DW}_{2}$ as a function of the applied SO field.

Thus, using the relation $2 \operatorname{atanh}(x)=\ln [(1+x) /(1-x)]$, the integral can be rewritten as

$$
I=2\left(X_{1}(t)-X_{2}(t)\right) \operatorname{csch}\left(\frac{X_{1}(t)-X_{2}(t)}{\Delta}\right),
$$

and, subsequently, Eq. (15) can be expressed as

$$
E_{\mathrm{exc}}^{1,2}=\frac{4 Q_{1} Q_{2} a_{0}^{2} A\left(X_{1}(t)-X_{2}(t)\right)}{\Delta^{2}} \operatorname{csch}\left(\frac{X_{1}(t)-X_{2}(t)}{\Delta}\right) .
$$

Summarizing all the exchange contributions of the system, taking into account Eqs. (9) and (19), Eq. (4) takes the functional form

$$
\begin{aligned}
E_{\mathrm{exc}}= & \frac{4 a_{0}^{2} A}{\Delta} \\
& +\frac{4 Q_{1} Q_{2} a_{0}^{2} A\left(X_{1}(t)-X_{2}(t)\right)}{\Delta^{2}} \operatorname{csch}\left(\frac{X_{1}(t)-X_{2}(t)}{\Delta}\right) .
\end{aligned}
$$

When both DWs have opposite topological charge $\left(Q_{1}+\right.$ $Q_{2}=0$ ), there exists a continuous transformation that permits both DWs to recombine giving rise to a uniform magnetic state. However, when $Q_{1}+Q_{2}=2$ [see Figs. 4(a) and 4(b)], there exists a region between both DWs whose polarization 
is antiparallel to $H_{\mathrm{SO}}$. As a consequence, the Zeeman energy $E_{\text {Zee }}$ pushes the DWs together in order to minimize the related energy [see Fig. 4(c)]. On the other hand, there also exists a repulsive interaction between $\mathrm{DW}_{1}$ and $\mathrm{DW}_{2}$ due to the exchange interaction $E_{\text {exc }}^{1,2}$ given by Eq. (19). This results in an expansion of the magnetic domain separating both DWs. Note that the central spins of each DW are antiparallel to each other, which implies that, to minimize the exchange energy, both magnetic textures have to be as far as possible from the other. The competition between these two forces, which leads to a stable distance among the two DWs, as shown in Fig. 4(b), can be expressed as

$$
\begin{aligned}
\Delta E= & \mu_{0} M_{\mathrm{s}} a_{0}^{2} H_{\mathrm{SO}}\left(X_{1}-X_{2}\right) \\
& +\frac{4 Q_{1} Q_{2} a_{0}^{2} A\left(X_{1}-X_{2}\right)}{\Delta^{2}} \operatorname{csch}\left(\frac{X_{1}-X_{2}}{\Delta}\right),
\end{aligned}
$$

where $\mu_{0}$ is the vacuum permeability and $M_{\mathrm{s}}$ represents the volumetric saturation magnetization [32]. Figure 4(d) shows the dependence of the Zeeman and exchange energies as a function of the relative distance between two DWs $\left(\mathrm{DW}_{1}\right.$ and $\mathrm{DW}_{2}$ ) with the same topological charge for an applied SO field of $20 \mathrm{mT}$. As the SO field and magnetic domain between the two DWs is antiparallel, the minimum energy corresponds to a zero distance between the DWs. The opposite happens with the exchange energy; the smaller the distance between the two DWs, the larger the exchange energy $(A>0)$. One can see [black dashed line in Fig. 4(d)] that when accounting for the exchange and the Zeeman energies, a global minimum appears which corresponds to a stable configuration distance between $\mathrm{DW}_{1}$ and $\mathrm{DW}_{2}$. A comparison between the stable distance among the two DWs as a function of the applied SO field extracted from numerical simulations and Eq. (21) is shown in Fig. 4(e). It can be observed that starting for the larger SO field $(60 \mathrm{mT})$, the stable distance increases as the $\mathrm{SO}$ field is reduced, meaning that the Zeeman force needs more extension of the magnetic domain to compensate the repulsion between the DWs.

\section{Energy flow from the collision of two DWs}

A fundamental question needs to be addressed: How much energy from the moving DWs is accessible to the external environment? The magnetic energy flow during the DWs collision involves the electron and lattice systems. To further quantify this process, we use a kinetic model where both local and nonlocal electron, phonon, and spin relaxations are included [42]. The dynamics of the electron and lattice vibrations (phonons) energies (expressed as their "effective quasiequilibrium temperatures" which can be a measurable quantity) are described by the two temperature model (TTM) [53] [Fig. 5(a)].

The fundamental assumption here is that the magnetic free energy flows directly into the electron system due to the metallic nature of $\mathrm{Mn}_{2} \mathrm{Au}$ [Fig. 5(a)].

$$
\begin{aligned}
C_{\mathrm{el}} \frac{d T_{\mathrm{el}}}{d t} & =-g_{\mathrm{el}-\mathrm{ph}}\left(T_{\mathrm{el}}-T_{\mathrm{ph}}\right)+\frac{\partial}{\partial x} \kappa \frac{\partial T_{\mathrm{el}}}{\partial x}+\dot{q} \\
C_{\mathrm{ph}} \frac{d T_{\mathrm{ph}}}{d t} & =g_{\mathrm{el}-\mathrm{ph}}\left(T_{\mathrm{el}}-T_{\mathrm{ph}}\right) .
\end{aligned}
$$

(a)
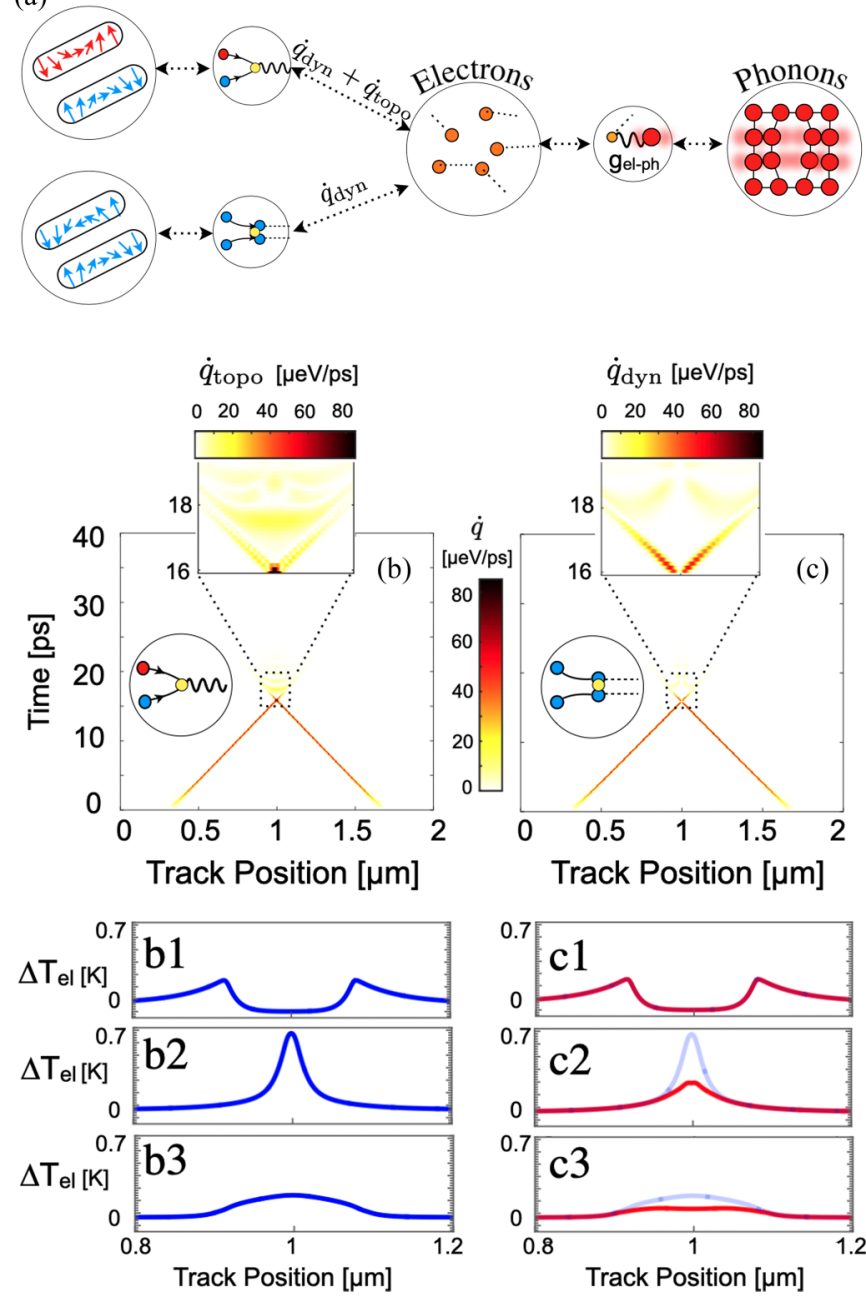

FIG. 5. (a) Stored energy at two domain walls (DWs) with opposite topology can be transferred to the electron system by colliding them. Besides $\dot{q}_{\mathrm{dyn}}$, an additional topologically-mediated transfer mechanism opens up, $\dot{q}_{\text {topo }}$, when the winding of the DWs are opposite. The electron and lattice are coupled via electron-phonon coupling, $g_{\text {el-ph }}$ (b) Color map of the spatiotemporal distribution of the rate of heat dissipation caused by the motion of the DWs. At the collision (zoomed in inset), an explosion of heat occurs due to the recombination of DWs. Subplots (b1)/(c1), (b2)/(c2), and (b3)/(c3) represent the spatial distribution of $\Delta T_{\mathrm{el}}$ along the track for three characteristic times: 14,16 , and 18 picoseconds, respectively.

The electron system receives an input of energy from the DW motion due to the magnetic friction (spin-Peltier effect [42]), spin-wave attenuation, and DW collision, all of them quantified by the Rayleigh dissipation functional $\dot{q}=\eta \dot{s}^{2}$, where $s$ represents the spin. $\eta=\mu_{\mathrm{s}} \alpha_{\mathrm{G}} / \gamma$, where $\gamma$ is the gyromagnetic ratio. Since the electron system has a much lower specific heat $\left(C_{\mathrm{el}}=\gamma_{\mathrm{el}} T_{\mathrm{el}}\right)$ than the lattice $\left(C_{\mathrm{ph}}\right)$, the electron system heats up almost instantaneously at a temperature that is larger than the lattice. The lattice heats only indirectly due to the coupling to the hot electrons via the electron-phonon coupling $g_{\text {el-ph }}$ [Fig. 5(a)]. The lateral thermal electron diffusion (defined by the parameter $\kappa$ ) is also included. We assume room temperature parameters [42]. The details of the electron and phonon temperature dynamics can 
(a)

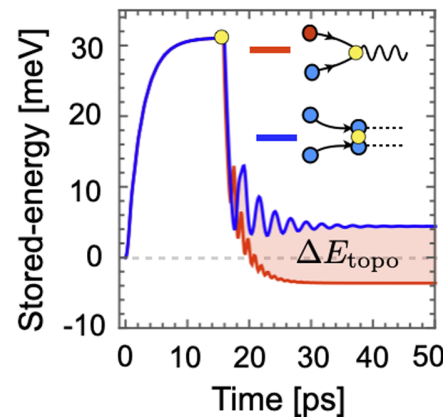

(b)

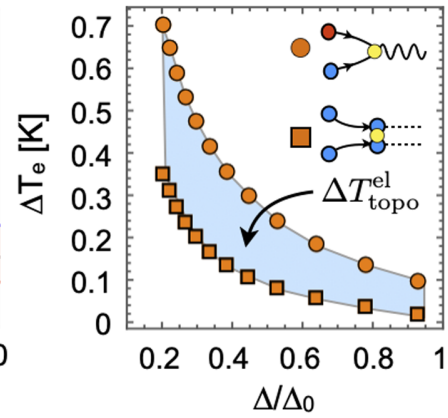

(c)

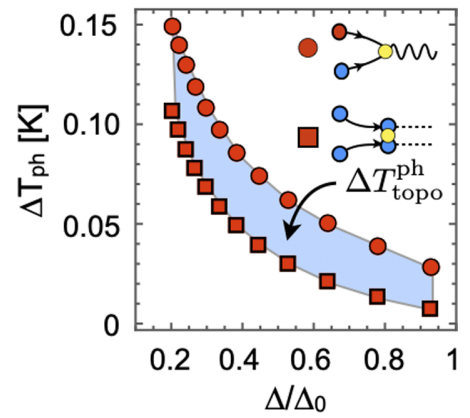

FIG. 6. (a) Time evolution of the stored energy at the DWs. Before domain wall (DW) collision (yellow circle), energy increases as the velocity of the DWs accelerates. After collision (yellow circle), for $Q_{1}=-Q_{2}$, there is a complete release of stored energy, while for $Q_{1}=Q_{2}$, a partial release of stored energy takes place. The difference of stored-energy release is defined as $\Delta E_{\text {topo }}=\Delta E\left(Q_{1},-Q_{1}\right)-\Delta E\left(Q_{1}, Q_{1}\right)$. (b) The maximum (at the collision) temperature increase of the electron system as a function of the reduced DW width, $\Delta / \Delta_{0}$. The shaded area defines the topological effect $\Delta T_{\text {topo }}^{\text {el }}=\Delta T_{\text {el }}\left(Q_{1},-Q_{1}\right)-\Delta T_{\mathrm{el}}\left(Q_{1}, Q_{1}\right)$. (c) The maximum (at the collision) temperature increase of the phonon system, as a function of the reduced DW width. The shaded area defines the topological effect $\Delta T_{\mathrm{topo}}^{\mathrm{ph}}=\Delta T_{\mathrm{ph}}\left(Q_{1},-Q_{1}\right)-\Delta T_{\mathrm{ph}}\left(Q_{1}, Q_{1}\right)$.

be visualized in Supplemental Material [48] for the two topological classes presented above.

Moving magnetic textures release energy to external medium by magnetic friction (spin-Peltier effect) [42]. For a stationary moving DW, the maximum heat is released at the DW center and the rate of its density $\dot{q}$ is defined by

$$
\dot{q}_{\text {dyn }, \text { stat }}=\frac{\mu_{\mathrm{s}}}{\gamma} \alpha_{\mathrm{G}}\left(\frac{v_{s}}{\Delta}\right)^{2} .
$$

We observe [Figs. 5(b) and 5(c)] that while the DWs move towards each other, their dissipation rates are equal, $\dot{q}_{1}=\dot{q}_{2}$, and closely follow Eq. (23). Before the collision [Figs. 5(b), 5(c), 5(b1), and 5(c1)], two well-defined electron temperature peaks can be clearly observed, corresponding to the DW positions. As DWs approach each other closer, their shapes are affected by their mutual interaction and individual topology. Spin-wave generation is also observed. Remarkably, DWs positions are clearly defined until the collision, marked by two separate $\Delta T_{\text {el }}$ peaks.

When $Q_{1}=Q_{2}$, at the instant of DWs collision, due to the repulsion effect, their positions never merge [visible as a white spot in the dissipated energy in the inset of Fig. 5(c)]. After the collision, DWs velocities change and the energy excess redistributes between different subsystems via spin wave emission, electron-phonon coupling, and lateral thermal conduction. This leads to a small energy peak [Fig. 5(c2)]. Since DWs remain well-defined separate entities during the entire dynamics, we identify this process as a purely dynamical energy release $\dot{q}_{\text {dyn }}$, the same as the spinPeltier effect in moving magnetic textures [42] but for a more complex situation.

On the contrary, when $Q_{1}=-Q_{2}$, at the moment of DW relativistic collision $[t=16 \mathrm{ps}$, Fig. 5(b2)], there is a large temperature boost [visible as a dark spot in the dissipated energy in the inset of Fig. 5(b)] which adds to the temperature increase from magnetic friction $\dot{q}_{\text {dyn }}$. Since this effect is caused by the annihilation of TMS subject to special topological rules, we call this process topological energy release $\left(\dot{q}_{\text {topo }}\right)$. Soon after $[t=18$ ps, Fig. 5(b3)], we again observe attenuation of $\Delta T_{\mathrm{el}}$ at the collision site due to the energy redistribution via electron-phonon coupling and lateral thermal conduction. On a longer timescale the breather disperses causing lateral spin waves which finally attenuate, passing the energy to electron and phonon systems.

The stored energy in the DW increases as the DW accelerates to reach their maximum velocity given by the SO field [Fig. 6(a)]. Interestingly, the dynamics of DW energies resembles that of the electric capacitor charging and discharging. Control of the amount of energy released into the medium is possible using high-energy AFM DWs. Since the self-energy of the individual DWs follows the special relativity Eq. (1), one can adjust it by modifying the width of the DW at the moment of the collision $\Delta_{c}$ [Figs. 6(b) and 6(c)]. The variation of the self-energy of the DWs before and after the collision, $\Delta E_{\text {col }}$, depends on both $\Delta_{\mathrm{c}} / \Delta_{0}$ and their relative winding numbers $Q_{1}, Q_{2}$. From our calculations, for relatively large velocities, close to $c$, the reduction of $\Delta$ can reach relative values of $\Delta_{\mathrm{c}} / \Delta_{0} \approx 0.2$. For the system parameters considered here, for collisions events with $Q_{1} Q_{2}<1$ occurring at the highest velocities (corresponding to SO field $=60 \mathrm{mT}$ ), we estimate the transferred energy $\Delta E_{\text {topo }} \sim 35 \mathrm{meV}$. In particular, we define topological energy variation in terms of the corresponding temperatures as $\Delta T_{\text {topo }}^{\mathrm{el}(\mathrm{ph})}=\Delta T_{\mathrm{el}(\mathrm{ph})}\left(Q_{1},-Q_{1}\right)-$ $\Delta T_{\mathrm{el}(\mathrm{ph})}\left(Q_{1}, Q_{1}\right)$, represented in Fig. 6 as a function of the DW width. We predict a measurable phonon temperature difference between the two topologically distinct DW collision processes, $\Delta T_{\text {topo }}^{\mathrm{ph}}=0.05 \mathrm{~K}$.

One can easily estimate the size of the energy release spot by noticing that the relaxation time of the spin dynamics (Supplemental Material [48]) is $\approx 3-4$ ps. For example, for the case considered here, $v_{s} \sim 42.4 \mathrm{~km} / \mathrm{s}$, the energy is released at a region of around 120-160 nm [Figs. 5(b1)/5(c1) to $5(\mathrm{~b} 3) / 5(\mathrm{c} 3)]$. Collisions at slower velocities permit us to narrow down the spot size, but at the same time the released energy will be smaller. Moreover, despite the intrinsic diffi- 
culties to measure magnetic signal in AFMs, the temperature traceability unveiled by our proposal opens the door to track experimentally the location of topological magnetic solitons and their interactions in AFMs.

\section{CONCLUSION}

We show that TMSs can be used as energy carriers, in particular AFM DWs can uptake, transport, and release energy. Our work allows us to unveil mechanisms of these processes. In a first step, we have demonstrated that relativistic kinematics of AFM TMS permits ultrafast energy uptake. This process is based on the relativistic DW contraction which allows up to a fivefold increase of the exchange energy present at the DW. Next, thanks to the soliton nature of AF DWs, they can propagate along the material allowing long-range energy transport. Finally, topologically conserved collisions of two DWs which allow for annihilation processes can serve as a transfer protocol to make use of the energy transported by the AF DWs in a fast manner.

The energy uptake, transport, and release mechanisms considered here are universal for any type of AFM TMS (skyrmions, vortices, etc.) and may also occur in ferromagnetic TMSs, if they could be accelerated to very high velocities avoiding the Walker breakdown. Recently, practical realization of relativistic kinematics in isolated magnetic solitons has been demonstrated [43]. Thus, extending those experiments to collisions of TMSs moving at relativistic speeds should be straightforward.
Our proposal also opens the door to ultrafast energy management at the nanoscale for future nanoelectronics. AFM TMS have the potential to become a new niche for energy transport devices based on electron's spin rather than on its charge. In comparison to other topological (nonmagnetic) defects, our approach avoids the inconvenience of overcoming any energy barrier for the particle-antiparticle recombination processes which are secured due to topological and energetic arguments. The energy release time is in the picosecond time scale and depends on inherent material properties, opening the door for further fine tuning of this intrinsic time.

\section{ACKNOWLEDGMENTS}

The work of R.M.O. was partially supported by the STSM Grants from the COST Action CA17123 Ultrafast optomagneto-electronics for nondissipative information technology. R.M.O. would like to thank Andrew Ramsey for useful discussions. U.A. and R.R.-E. acknowledge support from the Deutsche Forschungsgemeinschaft through SFB/TRR 227 "Ultrafast Spin Dynamics," Project A08. G.T. acknowledges the Grant-in-Aid for Scientific Research (B) (No. 17H02929) from the Japan Society for the Promotion of Science. O.C-F. acknowledges the financial support from Spanish Ministry of Science and Innovation under Grant No. PID2019108075RB-C31I00/AEI/10.13039/501100011033.
[1] M. Armand and J.-M. Tarascon, Building better batteries, Nature (London) 451, 652 (2008).

[2] B. Dieny et al., Opportunities and challenges for spintronics in the microelectronics industry, Nat. Electron. 3, 446 (2020).

[3] Z. Qiu, D. Hou, J. Barker, K. Yamamoto, O. Gomonay, and E. Saitoh, Spin colossal magnetoresistance in an antiferromagnetic insulator, Nat. Mater. 17, 577 (2018).

[4] S. S. Parkin, M. Hayashi, and L. Thomas, Magnetic domainwall racetrack memory, Science 320, 190 (2008).

[5] D. Allwood, G. Xiong, M. Cooke, C. Faulkner, D. Atkinson, N. Vernier, and R. Cowburn, Submicrometer ferromagnetic not gate and shift register, Science 296, 2003 (2002).

[6] T. An, V. I. Vasyuchka, K. Uchida, A. V. Chumak, K. Yamaguchi, K. Harii, J. Ohe, M. B. Jungfleisch, Y. Kajiwara, H. Adachi, B. Hillebrands, S. Maekawa, and E. Saitoh, Unidirectional spin-wave heat conveyer, Nat. Mater. 12, 549 (2013).

[7] E. Y. Vedmedenko and D. Altwein, Topologically Protected Magnetic Helix for All-Spin-Based Applications, Phys. Rev. Lett. 112, 017206 (2014).

[8] Y. Tserkovnyak and J. Xiao, Energy Storage Via Topological Spin Textures, Phys. Rev. Lett. 121, 127701 (2018).

[9] D. Jones, J. Zou, S. Zhang, and Y. Tserkovnyak, Energy storage in magnetic textures driven by vorticity flow, Phys. Rev. B 102, 140411(R) (2020).

[10] A. N. Bogdanov and U. K. Rößler, Chiral Symmetry Breaking in Magnetic Thin Films and Multilayers, Phys. Rev. Lett. 87, 037203 (2001).
[11] U. K. Roessler, A. Bogdanov, and C. Pfleiderer, Spontaneous skyrmion ground states in magnetic metals, Nature (London) 442, 797 (2006).

[12] K. Litzius, J. Leliaert, P. Bassirian, D. Rodrigues, S. Kromin, I. Lemesh, J. Zazvorka, K.-J. Lee, J. Mulkers, N. Kerber, D. Heinze, N. Keil, R. M. Reeve, M. Weigand, B. Van Waeyenberge, G. Schütz, K. Everschor-Sitte, G. S. D. Beach, and M. Kläui, The role of temperature and drive current in skyrmion dynamics, Nat. Electron. 3, 30 (2020).

[13] H.-B. Braun, Topological effects in nanomagnetism: from superparamagnetism to chiral quantum solitons, Adv. Phys. 61, 1 (2012).

[14] D. A. Allwood, G. Xiong, C. Faulkner, D. Atkinson, D. Petit, and R. Cowburn, Magnetic domain-wall logic, Science 309, 1688 (2005).

[15] S. Fukami, T. Suzuki, K. Nagahara, N. Ohshima, Y. Ozaki, S. Saito, R. Nebashi, N. Sakimura, H. Honjo, K. Mori et al., Low-current perpendicular domain wall motion cell for scalable high-speed MRAM, in 2009 Symposium on VLSI Technology (IEEE, 2009), pp. 230-231.

[16] V. Pribiag, I. Krivorotov, G. Fuchs, P. Braganca, O. Ozatay, J. Sankey, D. Ralph, and R. Buhrman, Magnetic vortex oscillator driven by de spin-polarized current, Nat. Phys. 3, 498 (2007).

[17] J. Grollier, D. Querlioz, and M. D. Stiles, Spintronic nanodevices for bioinspired computing, Proc. IEEE 104, 2024 (2016).

[18] J. Grollier, D. Querlioz, K. Camsari, K. Everschor-Sitte, S. Fukami, and M. D. Stiles, Neuromorphic spintronics, Nat. Electron. 3, 360 (2020). 
[19] A. Hubert and R. Schäfer, Magnetic Domains: The Analysis of Magnetic Microstructures (Springer, Berlin, 1998).

[20] N. L. Schryer and L. R. Walker, The motion of 180 domain walls in uniform dc magnetic fields, J. Appl. Phys. 45, 5406 (1974).

[21] J. C. Slonczewski, Current-driven excitation of magnetic multilayers, J. Magn. Magn. Mater. 159, L1 (1996).

[22] I. M. Miron, T. Moore, H. Szambolics, L. D. Buda-Prejbeanu, S. Auffret, B. Rodmacq, S. Pizzini, J. Vogel, M. Bonfim, A. Schuhl et al., Fast current-induced domain-wall motion controlled by the rashba effect, Nat. Mater. 10, 419 (2011).

[23] D.-S. Han, S.-K. Kim, J.-Y. Lee, S. J. Hermsdoerfer, H. Schultheiss, B. Leven, and B. Hillebrands, Magnetic domainwall motion by propagating spin waves, Appl. Phys. Lett. 94, 112502 (2009).

[24] X.-g. Wang, G.-h. Guo, Y.-z. Nie, G.-f. Zhang, and Z.-x. Li, Domain wall motion induced by the magnonic spin current, Phys. Rev. B 86, 054445 (2012).

[25] P. Yan, X. S. Wang, and X. R. Wang, All-Magnonic SpinTransfer Torque and Domain Wall Propagation, Phys. Rev. Lett. 107, 177207 (2011)

[26] J. Torrejon, G. Malinowski, M. Pelloux, R. Weil, A. Thiaville, J. Curiale, D. Lacour, F. Montaigne, and M. Hehn, Unidirectional Thermal Effects in Current-Induced Domain Wall Motion, Phys. Rev. Lett. 109, 106601 (2012).

[27] S. Selzer, U. Atxitia, U. Ritzmann, D. Hinzke, and U. Nowak, Inertia-Free Thermally Driven Domain-Wall Motion in Antiferromagnets, Phys. Rev. Lett. 117, 107201 (2016).

[28] E. V. Gomonay and V. M. Loktev, Spintronics of antiferromagnetic systems (Review Article), Low Temp. Phys. 40, 17 (2014).

[29] V. Baltz, A. Manchon, M. Tsoi, T. Moriyama, T. Ono, and Y. Tserkovnyak, Antiferromagnetic spintronics, Rev. Mod. Phys. 90, 015005 (2018).

[30] O. Gomonay, V. Baltz, A. Brataas, and Y. Tserkovnyak, Antiferromagnetic spin textures and dynamics, Nat. Phys. 14, 213 (2018).

[31] T. Shiino, S.-H. Oh, P. M. Haney, S.-W. Lee, G. Go, B.-G. Park, and K.-J. Lee, Antiferromagnetic Domain Wall Motion Driven by Spin-Orbit Torques, Phys. Rev. Lett. 117, 087203 (2016).

[32] R. M. Otxoa, P. E. Roy, R. Rama-Eiroa, J. Godinho, K. Y. Guslienko, and J. Wunderlich, Walker-like domain wall breakdown in layered antiferromagnets driven by staggered spin-orbit fields, Commun. Phys. 3, 190 (2020).

[33] A. Mougin, M. Cormier, J. P. Adam, P. J. Metaxas, and J. Ferré, Domain wall mobility, stability and Walker breakdown in magnetic nanowires, Europhys. Lett. 78, 57007 (2007).

[34] R. Hertel and C. M. Schneider, Exchange Explosions: Magnetization Dynamics During Vortex-Antivortex Annihilation, Phys. Rev. Lett. 97, 177202 (2006).

[35] G. Tatara and R. M. Otxoa de Zuazola, Collective coordinate study of spin-wave emission from a dynamic domain wall, Phys. Rev. B 101, 224425 (2020).

[36] R. Hertel, Ultrafast domain wall dynamics in magnetic nanotubes and nanowires, J. Phys.: Condens. Matter 28, 483002 (2016).

[37] F. D. M. Haldane, Nonlinear Field Theory of Large-Spin Heisenberg Antiferromagnets: Semiclassically Quantized Solitons of the One-Dimensional Easy-Axis Néel State, Phys. Rev. Lett. 50, 1153 (1983).
[38] S. M. Mohseni, S. R. Sani, J. Persson, T. N. A. Nguyen, S. Chung, Y. Pogoryelov, P. K. Muduli, E. Iacocca, A. Eklund, R. K. Dumas, S. Bonetti, A. Deac, M. A. Hoefer, and J. Åkerman, Spin torque-generated magnetic droplet solitons, Science 339, 1295 (2013).

[39] R. Rajaraman, Solitons and Instantons (North-Holland, Amsterdam, 1982).

[40] K. Olejník, T. Seifert, Z. Kašpar, V. Novák, P. Wadley, R. P. Campion, M. Baumgartner, P. Gambardella, P. Němec, J. Wunderlich, J. Sinova, P. Kužel, M. Müller, T. Kampfrath, and T. Jungwirth, Terahertz electrical writing speed in an antiferromagnetic memory, Sci. Adv. 4, eaar3566 (2018).

[41] Z. Kašpar, M. Surýnek, J. Zubáč, F. Krizek, V. Novák, R. P. Campion, M. S. Wörnle, P. Gambardella, X. Marti, P. Němec, K. W. Edmonds, S. Reimers, O. J. Amin, F. Maccherozzi, S. S. Dhesi, P. Wadley, J. Wunderlich, K. Olejník, and T. Jungwirth, Quenching of an antiferromagnet into high resistivity states using electrical or ultrashort optical pulses, Nat. Electron. 4, 30 (2021)

[42] R. M. Otxoa, U. Atxitia, P. E. Roy, and O. Chubykalo-Fesenko, Giant localised spin-Peltier effect due to ultrafast domain wall motion in antiferromagnetic metals, Commun. Phys. 3, 31 (2020).

[43] L. Caretta, S.-H. Oh, T. Fakhrul, D.-K. Lee, B. H. Lee, S. K. Kim, C. A. Ross, K.-J. Lee, and G. S. D. Beach, Relativistic kinematics of a magnetic soliton, Science 370, 1438 (2020).

[44] J. Zang, V. Cros, and A. Hoffmann, Topology in Magnetism (Springer, Cham, Switzerland, 2018), Vol. 192.

[45] V. M. T. S. Barthem, C. V. Colin, H. Mayaffre, M.-H. Julien, and $\mathrm{D}$. Givord, Revealing the properties of $\mathrm{Mn}_{2} \mathrm{Au}$ for antiferromagnetic spintronics, Nat. Commun. 4, 2892 (2013).

[46] A. Manchon, J. Železný, I. M. Miron, T. Jungwirth, J. Sinova, A. Thiaville, K. Garello, and P. Gambardella, Current-induced spin-orbit torques in ferromagnetic and antiferromagnetic systems, Rev. Mod. Phys. 91, 035004 (2019).

[47] S. Ganichev, Spin-galvanic effect and spin orientation by current in non-magnetic semiconductors, Int. J. Mod. Phys. B 22, 1 (2008).

[48] See Supplemental Material at http://link.aps.org/supplemental/ 10.1103/PhysRevResearch.3.043069 for crystallographic structure and domain wall distribution, domain walls collision evolution depending on the relative topological number, and breather characterization.

[49] P. E. Roy, R. M. Otxoa, and J. Wunderlich, Robust picosecond writing of a layered antiferromagnet by staggered spin-orbit fields, Phys. Rev. B 94, 014439 (2016).

[50] A. B. Shick, S. Khmelevskyi, O. N. Mryasov, J. Wunderlich, and T. Jungwirth, Spin-orbit coupling induced anisotropy effects in bimetallic antiferromagnets: A route towards antiferromagnetic spintronics, Phys. Rev. B 81, 212409 (2010).

[51] G. Tatara, C. A. Akosa, and R. M. Otxoa de Zuazola, Magnon pair emission from a relativistic domain wall in antiferromagnets, Phys. Rev. Research 2, 043226 (2020).

[52] I. S. Gradshteyn and I. M. Ryzhik, Table of Integrals, Series, and Products (Academic Press, Waltham, MA, 2015) Vol. 4, p. 173.

[53] M. I. Kaganov, I. M. Lifshitz, and L. V. Tanatarov, Relaxation between electrons and crystalline lattices, JETP 173 (1957). 